\title{
Análise da Fadiga Muscular Localizada em Atletas e Sedentários Através de Parâmetros de Freqüência do Sinal Eletromiográfico
}

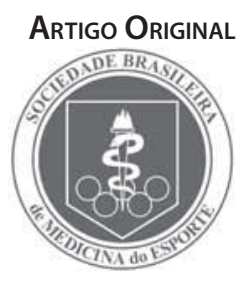

\section{Analysis of Localized Muscular Fatigue in Athletes and Sedentary Subjects through Frequency Parameters of Electromyographic Signal}

Marcelo Cláudio Amaral Santos ${ }^{1,2}$ Tatiana Adamov Semeghuini² Fábio Mícolis de Azevedo 3,4 Diego Basile Colugnati ${ }^{5}$

Rúben de Faria Negrão Filho ${ }^{3}$ Neri Alves ${ }^{3}$

Ricardo Mário Arida

1. Programa de Pós-Graduação em Fisiologia do Exercício - Escola Paulista de Medicina - Unifesp

- São Paulo, SP - Brasil.

2. Universidade do Oeste Paulista

- Faculdade de Fisioterapia

- Presidente Prudente, SP- Brasil.

3. Laboratório de Fisioterapia

Aplicada ao Movimento Humano

- LAFAMH / FCT - Unesp - Campus de Presidente Prudente, SP - Brasil. 4. Instituto de Ensino e Pesquisa Movimentare Fisioterapia e

Reabilitação - Presidente Prudente, SP - Brasil.

5. Disciplina de Neurologia Experimental- Escola Paulista de Medicina - Unifesp - São Paulo, SP - Brasil.

\section{Endereço para correspondência:} Prof. Dr. Fábio Mícolis de Azevedo, Rua Coriolano Gomes Palmeira, 160 19013-790 - Presidente Prudente, SP. Tels.: (18) 8129-0819 - 3222-2464.

E-mail:micolis@uol.com.br

Submetido em 13/12/2007

Versão final recebida em 28/04/2008 Aceito em 05/07/2008

\begin{abstract}
RESUMO
Embora a análise no domínio da freqüência do sinal eletromiográfico (EMG) seja empregada na caracterização do processo de fadiga muscular localizada, sua aplicação, especificamente a da freqüência mediana $\left(F_{\text {med }}\right)$, é pouco explorada no âmbito esportivo. O objetivo do presente estudo foi verificar a viabilidade da aplicação do sinal EMG, através de sua análise no domínio da freqüência, como parâmetro para determinação e diferenciação no comportamento da fadiga muscular localizada. Dois grupos de sujeitos, um caracterizado como atletas $(n=12)$ e outro como sedentários $(n=12)$, foram submetidos a análises baseadas em procedimentos executados em três diferentes situações experimentais, todos envolvendo a modalidade de exercício isométrico: i) teste máximo para determinação da contração isométrica voluntária máxima (CIVM); ii) teste de fadiga, sustentado por 35 seg. a 80\% da CIVM; iii) teste de recuperação, sustentado por 10 seg. a $80 \%$ da CIVM; neste ultimo foi monitorado o comportamento da $F_{\text {med }}$ nos três primeiros $\left(\mathbf{F}_{\text {medi }}\right)$ e três últimos segundos $\left(\mathbf{F}_{\text {medf }}\right)$ do sinal $E M G$ no músculo tibial anterior durante o teste de fadiga. Durante os 10 segundos do teste de recuperação foi calculada a $F_{\text {med }}$ referente a todo o período $\left(\boldsymbol{F}_{\text {medr }}\right)$. parâmetro utilizado no cálculo do índice de recuperação muscular (IRM). Os resultados apontam que a $\mathbf{F}_{\text {medf }}$ apresentou valor menor em relação à $\mathbf{F}_{\text {medi }}$ em ambos os grupos $(p<0,05)$. Quando comparado com o grupo de sedentários, o grupo de atletas apresentou valores maiores de $\mathbf{F}_{\text {medi }}$ e $\mathbf{F}_{\text {medf }}(p<0,05)$. O valor médio e desvio-padrão do IRM para o grupo de atletas foram de $62,1 \% \pm 28,7$ e, para o grupo de sedentários, de $55,2 \% \pm 27,8$ ( $p>0,05)$. Dessa forma, os resultados apresentados neste estudo permitem inferir a viabilidade na aplicação de parâmetros no domínio da freqüência do sinal EMG para a determinação e diferenciação do comportamento da fadiga muscular localizada.
\end{abstract}

Palavras-chave: biomecânica, eletromiografia, fadiga muscular, músculo tibial anterior, processamento digital de sinal, esporte.

\begin{abstract}
Although the analysis in the frequency domain of the Electromyographic Signal (EMG) has been used in the characterization of the localized muscular fatigue process, its application, specifically the Median Frequency (MF), is rarely explored in sports. The objective of this study was to verify the viability of the EMG signal application, through its frequency domain analysis, as a parameter for determination and differentiation of the behavior of localized muscle fatigue. Two groups of subjects, one characterized as athletes $(n=12)$ and the other as sedentary $(n=12)$, were submitted to analysis based on procedures from three different experimental situations, all involving isometric exercise modality: i) maximum test for determination of the Maximum Voluntary Isometric Contraction (MVIC); ii) fatigue test, 35 sec. sustained load of $80 \%$ of MVIC; iii) recovery test, 10 sec. sustained load of $80 \%$ of MVIC. In the latter, the MF behavior in the three first $\left(F_{\text {medi }}\right)$ and three last $\left(F_{\text {medf }}\right)$ seconds of the EMG signal of tibialis anterior muscle during the fatigue test have been monitored. During the 10 seconds of the recovery test, MF was calculated regarding the whole period ( $\left.F_{\text {medr }}\right)$; this parameter was used to calculate the Muscular Recovery Index (MRI). The results showed that $F_{\text {medf }}$ presented lower value in relation to $F_{\text {medi }}$ in both groups $(p<0.05)$. Additionally, the $F_{\text {medi }}$ and $F_{\text {medf }}$ values for the athlete group were higher in comparison to the sedentary group $(p<0.05)$. The MRI mean value and standard deviation for the athlete group were $62.1 \% \pm 28.7$ and for the sedentary group was $55.2 \% \pm 27.8(p>0.05)$. Therefore, the results presented in this study allow inferring the viability in the application of the frequency domain parameters of the EMG signal for the determination and differentiation of localized muscle fatigue behavior.
\end{abstract}

Keywords: biomechanics, electromyography, muscle fatigue, tibialis anterior muscle, digital signal processing, sports.

\section{INTRODUÇÃO}

A fadiga muscular localizada pode ser caracterizada como o declínio do desempenho muscular durante o exercício. Usualmente, a deterioração do desempenho mecânico é o parâmetro que os especialistas adotam para determinar um "ponto de fadiga" no qual o músculo não é mais capaz de sustentar um nível de força desejado ${ }^{(1-4)}$. Esse fenômeno tem sido amplamente estudado, mas sua etiologia ainda não foi totalmente elucidada. Entretanto, sabe-se que o comportamento dos padrões de impulsos neuromusculares que ocorrem durante as contrações musculares caracteriza um dos fatores centrais de sua causa ${ }^{(5)}$.

Estudos em biomecânica têm abordado amplamente o fenômeno 
da fadiga através da progressiva e contínua atividade muscular voluntária que leva a alteração do comportamento do sinal eletromiográfico (EMG) captado no músculo exercitado(6-8). Entende-se que essa alteração se deve, principalmente, aos processos fisiológicos ligados ao recrutamento por soma das unidades motoras e alteração na velocidade de condução do potencial de ação ao longo da fibra muscular. Esse último fundamenta e caracteriza a análise do espectro de freqüência do sinal EMG ${ }^{(9)}$.

Nesse contexto, sugere-se que a diminuição das taxas de disparo das unidades motoras contribuirá para que ocorram mudanças na densidade do espectro de potência do sinal EMG, caracterizando a alteração do estado fisiológico muscular. Em situações de fadiga são observados simultaneamente aumento nos componentes de baixa freqüência e, de forma mais discreta, diminuição nos de alta freqüência(9-11).

Dessa forma, a constatação de que o fenômeno da fadiga muscular localizada altera certas faixas de freqüência do sinal EMG determina a possibilidade de se detectar a instalação desse processo através da observação de indicadores relacionados à densidade de seu espectro de freqüência ${ }^{(12)}$. O indicador mais utilizado é a freqüência mediana do espectro de freqüência do sinal EMG, pois em sua aplicação reside maior respaldo científico(13).

Algumas diferenças já foram relatadas e descritas no que se refere à taxa de queda da freqüência mediana durante o exercício ${ }^{(4,8)}$. De maneira ampla, acredita-se que o comportamento da freqüência mediana de um sujeito treinado é diferente do de um sedentário. Basicamente, o treinado deve apresentar queda mais lenta desse parâmetro durante o tempo de exercício quando comparado com um sujeito sedentário ${ }^{(12)}$.

Nesse sentido, outros estudos também observam o índice de recuperação muscular. Basicamente, o índice de recuperação muscular pode ser entendido como a capacidade do músculo em apresentar um valor de freqüência mediana próximo ao observado no repouso, após ser submetido a um teste de fadiga. A análise desse parâmetro sugere que o músculo do sujeito treinado tem maior capacidade de se recuperar, após ser submetido a um exercício que induz a fadiga muscular localizada, que o músculo de um sujeito sedentário(14).

Portanto, o sinal EMG e, especificamente, os parâmetros descritos acima caracterizam uma importante fonte de dados quantitativos e objetivos a respeito da capacidade de resistência muscular do sujeito. Configura-se, dessa forma, um método que apresenta grande potencial de aplicação na área do treinamento físico visando o desempenho ou a saúde. Entretanto a quantidade de investimentos científicos na busca de validação de metodologias biomecânicas, como o uso do sinal EMG, para monitoração do processo de fadiga muscular localizada, ainda é escassa e também controversa. Fator que torna essa temática desafiadora, uma vez que as prescrições de exercícios que objetivam as adaptações de resistência são baseadas em dados que não refletem as condições fisiológicas musculares in loco ${ }^{(15)}$.

Sendo assim, o presente estudo teve por objetivo verificar a viabilidade na aplicação do sinal EMG, através de sua análise no domínio da freqüência, como parâmetro para determinação e diferenciação no comportamento da fadiga muscular localizada no músculo tibial anterior de sujeitos atletas e sedentários.

\section{MÉTODOS}

\section{Grupo amostral}

Foram selecionados 12 sujeitos, considerados sedentários ${ }^{(16)}$, do gênero masculino com, idade de $22 \pm 2,17$ anos, massa de 74,70 \pm $10,60 \mathrm{~kg}$ e altura de 1,77 $\pm 0,05 \mathrm{~m}$. Também foram selecionados 12 atletas profissionais, jogadores de futebol, com idade de $20 \pm 1,05$ anos, massa de 71,74 $\pm 6,24 \mathrm{~kg}$ e altura de 1,79 $\pm 0,04 \mathrm{~m}$. Na amostra foram excluídos os sujeitos que apresentaram alguma desordem neuromuscular e/ou articular, no membro inferior dominante, em período inferior a 18 meses. O membro dominante foi determinado baseando-se na preferência do sujeito em realizar um gesto de chute ${ }^{(17,18)}$.

Antes da realização dos testes, todos os sujeitos selecionados visitaram o laboratório para ser orientados sobre sua participação no estudo e procedimentos adotados. Após os esclarecimentos, eles assinaram um termo de consentimento livre e esclarecido. Os procedimentos utilizados neste estudo foram analisados pela comissão de ética local, da Universidade do Oeste Paulista-Unoeste, obtendo parecer favorável através do processo n 196/1996.

\section{Instrumentação}

Para a aquisição do sinal eletromiográfico (EMG) foi utilizado um eletrodo ativo do tipo "encapsulado" composto por duas barras de $\mathrm{AgCl}$ com $10 \mathrm{~mm}$ de comprimento e $1 \mathrm{~mm}$ de largura (marca Lynx $x^{\oplus}$ Tecnologia Eletrônica Ltda.). Esse tipo de eletrodo carrega em seu corpo um circuito pré-amplificador com ganho de 20 vezes, CMRR (common mode rejection ratio) maior que $80 \mathrm{~dB}$ e impedância de $1.012 \Omega$. O ponto motor foi localizado na região do ventre muscular do músculo tibial anterior do sujeito. Utilizou-se um aparelho de eletroestimulação (modelo Neurodyn ${ }^{\circledR}$, marca Ibramed ${ }^{\circledast}$ ) e um eletrodo tipo "caneta". Após a localização e marcação do ponto, foram feitas tricotomia, abrasão e limpeza da região. O eletrodo foi fixado $3 \mathrm{~cm}$ abaixo do ponto motor localizado(10).

O sinal EMG foi captado em um módulo condicionador de sinais (marca Lynx ${ }^{\oplus}$, modelo MCS1000 ${ }^{\circledR}$ ). Nesse módulo, um canal foi configurado com um filtro analógico de $4^{a}$ ordem, tipo Butterworth, passa-baixa com freqüência de corte de $500 \mathrm{~Hz}$ e um filtro passa-alta com freqüência de corte de $20 \mathrm{~Hz}$. O ganho final no canal foi de 1.000 vezes.

A monitoração da força aplicada pelo sujeito, durante o exercício isométrico, foi realizada através de uma célula de carga para ensaios de tensão de tração e compressão (marca Kratos ${ }^{\circledast}$, modelo MM ${ }^{\circledast}$ ). Um canal no condicionador de sinais foi configurado para a aquisição simultânea do sinal proveniente da célula de carga. Neste canal foi habilitado um filtro analógico de $4^{\mathrm{a}}$ ordem, tipo Butterworth, passabaixa de $100 \mathrm{~Hz}$. Tanto o canal para aquisição do sinal EMG quanto o de instrumentação foram configurados para uma freqüência de amostragem igual a $2.000 \mathrm{~Hz}$.

A aquisição e o armazenamento dos sinais em arquivos de dados foram feitos através do software Bioinspector v.1.8 (Lynx $\left.x^{\circledast}\right)$.

\section{Procedimentos experimentais}

Inicialmente, o sujeito foi posicionado em um banco, com ajustes para apoio e estabilização do tronco, quadril e coxa (marca EnrafNonius ${ }^{\circledast}$, modelo $E N$-Tree ${ }^{\circledast}$ ), obedecendo ao seguinte posicionamento: i) quadril em 90 graus de flexão; ii) joelho em flexão de 40 graus; iii) tornozelo em flexão plantar de 40 graus $^{(19-21)}$. A célula de carga foi acoplada a um dispositivo fixado na linha do primeiro metatarso do membro dominante e ao solo. Após o posicionamento do sujeito, os testes para coleta dos dados foram executados. Foram três etapas: i) teste máximo - determinação da contração isométrica voluntária máxima (CIVM); ii) teste de fadiga - execução do exercício sustentado por 35 segundos a 80\% da CIVM; iii) teste de recuperação - execução do exercício sustentado por 10 segundos a 80\% da CIVM.

Para determinar a CIVM, o sujeito realizou três contrações sustentadas por seis segundos com um descanso de 10 minutos entre as tentativas. Foram desconsiderados da análise os dois primeiros e dois últimos segundos coletados. Assim, considerou-se como CIVM a média geral dos dados obtidos nesse intervalo para as três contrações.

Os testes de fadiga e de recuperação foram executados respeitandose um intervalo mínimo de 48 horas do teste para determinação da CIVM do sujeito. No teste de fadiga, o sujeito foi orientado a sustentar por 35 segundos uma contração isométrica do músculo tibial anterior, a 80\% da 
CIVM, tendo como feedback visual a intensidade da força externa monitorada pela célula de carga. Após dois minutos de descanso, o sujeito realizou novamente uma contração isométrica sustentada por 10 segundos, denominada de teste de recuperação. O sinal EMG do músculo tibial anterior foi captado somente no teste de fadiga e de recuperação.

\section{Processamento dos dados}

Os sinais EMG captados no teste de fadiga e de recuperação foram processados através de um algoritmo especifico desenvolvido em ambiente MatLab ${ }^{\circledast}$. Na primeira fase de processamento aplicou-se ao sinal EMG, de ambos os testes, um filtro digital passa-banda tipo Butterworth de $4^{\mathrm{a}}$ ordem e freqüência de corte entre 20 e $500 \mathrm{~Hz}$.

Foram selecionados para análise os três primeiros e três últimos segundos do sinal EMG provenientes dos testes de fadiga. Os dados contidos nesses períodos foram aplicados à tranformada discreta de Fourier (TDF) para obtenção de suas respectivas densidades espectrais de potência. A freqüência mediana, definida como a freqüência que divide o espectro em duas áreas iguais, foi calculada para ambos os períodos. Constituíram os dois primeiros parâmetros extraídos do sinal EMG: i) freqüência mediana inicial $\left(\mathbf{F}_{\text {medi }}\right)$; ii) freqüência mediana final $\left(\mathbf{F}_{\text {medf }}\right)$.

A freqüência mediana dos testes de recuperação também foi calculada a partir do período total de 10 segundos de sinal EMG captado, caracterizando o terceiro parâmetro denominado de freqüência mediana de recuperação $\left(\mathbf{F}_{\text {medr }}\right)$.

Após a obtenção dos três parâmetros do sinal EMG descritos acima, foi calculado o índice de recuperação muscular (IRM), através da equação $1^{(14)}$.

$$
\text { IRM }=\frac{F_{\text {medr }} \quad F_{\text {medf }}}{F_{\text {medi }} F_{\text {medf }}} \sum 100
$$

Onde:

IRM - Índice de recuperação muscular;

$F_{\text {medi }}$ - Freqüência mediana inicial (teste de 35 segundos);

$F_{\text {medf }}$ - Freqüência mediana final (teste de 35 segundos);

$F_{\text {medr }}$ - Freqüência mediana de recuperação (teste de 10 segundos).

Para o tratamento estatístico dos dados, inicialmente, um teste de normalidade foi aplicado aos valores da $F_{\text {medir }} F_{\text {medf }}$ e do IRM. Utilizou-se, nesse caso, o teste de Shapiro-Wilks, que observa a correlação entre a amostra dos dados com seu correspondente escore normal, dado pelo valor de W. Assim, as comparações realizadas entre as variáveis de interesse foram estabelecidas da seguinte forma: i) os dados que apresentaram distribuição normal foram submetidos à análise de variância através da ANOVA com dois fatores (two-way). Neste caso foi utilizado o teste post hoc de Tukey; ii) aos dados que não apresentaram distribuição normal aplicou-se o teste de Mann-Whitney. Entende-se nestes casos que as condições exigidas pela análise de variância estão comprometidas. Para todos os testes foi considerado nível de significância de 5\%(22).

Os dados também foram analisados através de seu coeficiente de variação $(\mathrm{CV})$, definido como sendo o desvio-padrão da amostra expresso em percentagem de seu valor médio(22).

\section{RESULTADOS}

\section{Análise da freqüência mediana no início $\left(F_{\text {medi }}\right)$ e no final $\left(F_{\text {medf }}\right)$ do teste de fadiga}

A tabela 1 apresenta os valores médios da $\mathbf{F}_{\text {medi }}$ e $\mathbf{F}_{\text {medf }}$ dos grupos de atletas e sedentários e os respectivos resultados da análise estatística dos dados. Verifica-se que, em ambos os grupos, a $\mathbf{F}_{\text {medf }}$ apresentou valor significativamente menor que à $\mathbf{F}_{\text {medi }}$. Também se observa que os valores das $\mathbf{F}_{\text {medi }}$ e $\mathbf{F}_{\text {medf }}$ para o grupo de atletas foram maiores, com diferença significativa, em comparação com grupo sedentário. Entretanto, não foram encontradas diferenças significativas entre a $\mathbf{F}_{\text {medi }}$ do grupo de sedentários comparado com a $\mathbf{F}_{\text {medf }}$ do grupo de atletas.

Tabela 1. Valores médios e desvio-padrão da $\mathbf{F}_{\text {medi }}$ e $\mathbf{F}_{\text {medf }}$ para os grupos de sedentários $(n=12)$ e atletas $(n=12)$. Comparação das médias através do teste de variância ANOVA para dois fatores (sig. 5\%)

\begin{tabular}{c|c|c|c}
\hline \multirow{2}{*}{ Momentos } & \multicolumn{2}{|c|}{ Grupos } & \\
\cline { 2 - 4 } & Atletas & Sedentários & $\mathbf{p}$ \\
\hline$F_{\text {medi }}$ & $141,1^{\mathbf{a}, \mathbf{b}, \mathbf{c}} \pm 18,1$ & $118,8^{\mathfrak{b}} \pm 25,6$ & $<0,05$ \\
\hline$F_{\text {medf }}$ & $114,2^{\mathbf{b}} \pm 16,5$ & $90,2 \pm 18,6$ & $<0,05$ \\
\hline$p$ & $<0,05$ & $<0,05$ & \\
\hline
\end{tabular}

A Diferença significativa com relação a $\mathbf{F}_{\text {medf }}$ no grupo atletas.

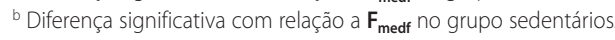

c Diferença significativa com relação a $\mathbf{F}_{\text {medi }}$ no grupo sedentários.

Uma análise descritiva dos dados, em relação aos seus coeficientes de variação (CV) demonstra que os valores calculados para a $\mathbf{F}_{\text {medi }}$ e $\mathbf{F}_{\text {medf }}$ do grupo de atletas, respectivamente, 12,8\% e 14,4\%, foram menores que os valores calculados para a $\mathbf{F}_{\text {medi }}$ e $\mathbf{F}_{\text {medf }}$ do grupo de sedentários, respectivamente, 21,5\% e 20\%.

\section{Índice de recuperação muscular}

Através dos testes de recuperação, obteve-se a $\mathbf{F}_{\text {medr }}$ utilizada no cálculo do índice de recuperação muscular (IRM) dos sujeitos componentes do grupo de atletas e de sedentários. O valor médio e desviopadrão do IRM para o grupo de atletas foi de 62,1\% $\pm 28,7$ e, para o de sedentários, de 55,2\% \pm 27,8. Na comparação dos valores médios do IRM entre os grupos, através do teste de Mann-Whitney, não se observou diferença significativa $(p>0,05)$.

Apesar de o teste estatístico não demonstrar diferença significativa, a análise descritiva dos dados apresenta a tendência de IRM maior para o grupo de atletas em comparação com o de sedentários. Os valores do coeficiente de variação para o IRM do grupo de atletas e sedentários foram de $46,2 \%$ e $50,3 \%$, respectivamente.

\section{DISCUSSÃO}

Inicialmente, é importante apresentar uma reflexão generalista em relação às delimitações e controle do erro experimental. Todo o procedimento de coleta de dados foi realizado em ambiente e em situação incomuns aos sujeitos, a lembrar: i) as instruções a que o sujeito deveria atender e a atenção às informações de feedback durante o teste de fadiga e recuperação; ii) o posicionamento dos sujeitos no equipamento de exercício; iii) a fixação dos eletrodos sobre a pele. Dadas essas restrições, pode-se ter condicionado algum tipo de alteração nas variáveis monitoradas, o que é denominado de efeito retroativo. Para minimizar esse efeito, ofereceu-se a oportunidade de o sujeito visitar o ambiente do laboratório para se familiarizar com os procedimentos. Esse procedimento é comum na área temática de que trata o presente estudo(23,24).

Com relação ao protocolo de exercício, observou-se que a sustentação por 35 segundos de 80\% da CIVM foi suficiente para a indução da fadiga muscular localizada no músculo tibial anterior. Fundamenta-se essa afirmação através da observação da diminuição nos valores da $\mathbf{F}_{\text {medf }}$ em relação a $\mathbf{F}_{\text {medi }}$ a ambos os grupos. Esse comportamento é amplamente reportado na literatura como indicativo de que o músculo está trabalhando em regime de fadiga ${ }^{(4,14,25)}$.

Observa-se também que a diferença entre a $\mathbf{F}_{\text {medi }}$ e a $\mathbf{F}_{\text {medf }}$ no grupo de sedentários foi mais acentuada se comparada com a de atletas. 0 presente estudo não apresenta os ajustes lineares que caracterizam esse comportamento ao longo dos 35 segundos de contração muscular. Entretanto, pode-se sugerir que a taxa de queda da freqüência mediana do sinal 
EMG foi maior no grupo composto por sujeitos sedentários se comparado com o de atletas. A taxa de queda da freqüência mediana é um indicador bastante utilizado para a determinação de parâmetros mais complexos ligados à monitoração da fadiga muscular localizada como, por exemplo, o índice de fadiga e do limiar de fadiga eletromiográfico ${ }^{(3,4,15)}$.

Dentro desse contexto, a análise do IRM do grupo de atletas demonstra tendência de esses sujeitos apresentarem maior capacidade de recuperação muscular após o exercício realizado em regime de fadiga. Sugere-se que esse comportamento possa estar relacionado à maior capacidade do sistema muscular dos atletas em remover os catabólicos responsáveis pela alteração do sinal EMG, que são produzidos durante o trabalho muscular em regime de fadiga. Nesse sentido, alguns autores relacionam a queda da freqüência mediana do sinal EMG, durante o exercício, a aumento na concentração de acido láctico no meio extracelular do tecido muscular. Teoricamente, o aumento na concentração de ácido láctico leva à diminuição do pH extracelular, fator que está relacionado com a diminuição na velocidade de condução do potencial de ação através da fibra muscular ${ }^{(8)}$. Entretanto, é importante ressaltar que a metodologia para determinação do IRM não goza de unanimidade dentro da literatura com relação, principalmente, a sua reprodutibilidade. Fator observado neste estudo através dos altos valores do coeficiente de variação calculados para o IRM de ambos os grupos ${ }^{(14)}$.

A literatura refere-se a outros fatores também relacionados com a manifestação da fadiga muscular localizada, como, por exemplo: i) o ritmo circadiano ${ }^{(26)}$; ii) aspectos psicológicos ${ }^{(27,28)}$ que por sua natureza constituem uma variável subjetiva e, por conseqüência, de difícil controle; iii) estratégias de controle do sistema nervoso central(4).

Em relação aos $C V$ de ambos os grupos, nota-se variação menor para o grupo de atletas em comparação com o de sedentários. Isso sugere que os atletas apresentam comportamento mais homogêneo da freqüência mediana, fator que se apresenta bem descrito na literatura e que pode estar relacionado tanto com o nível quanto com a especificidade de treinamento a que esses sujeitos são submetidos ${ }^{(8,29,30)}$.

De forma geral, os resultados apresentados neste estudo permitem inferir a viabilidade na aplicação de métodos em biomecânica para a

\section{REFERÊNCIAS BIBLIOGRÁFICAS}

1. Merletti R, Lo Conte LR. Surface EMG Signal processing during isometric contractions. J Electromyogr Kinesiol 1997;7:241-50

2. Merletti R, Knaflitz M, De Luca CJ. Electrically evoked myoelectric signals. Crit Rev Biomed Eng 1992;19:293-340

3. Merletti R, Lo Conte RL, Orizo C. Indices of muscle fatigue. J Electromyogr Kinesiol 1991;20:33-1.

4. Merletti R. Electromyography: physiology, engineering, and noninvasive aplications. Italy: Philip Paker, 2004.

5. Edwards RH, Hill DK, Jones DA, Merton PA. Fatigue of long duration in human skeletal muscle after exercise. J Physiol 1997;272:769-78.

6. Moritani T, Kimura T, Hamada T, Nagai N. Electrophysiology and kinesiology for health and disease. J Electromyogr Kinesiol 2005;15:240-55.

7. Moritani T, Yoshitake Y. 1998 ISEK Congress keynote lecture: the use of electromyography in applied physiology. J Electromyogr Kinesiol 1998;8:363-81.

8. Moritani T, Takaishi T, Matsumoto T. Determination of maximal power output at neuromuscular fatigue threshold. J Appl Physiol 1993;74:1729-34.

9. Moritani T, Nagata E, Muro M. Electromyographyc manifestation of muscular fatigue. Med Phys Fitness 1982;14:198-202.

10. Basmajian J, De Luca CJ. Muscles alive: their functions revealed by eletromyography. 5th ed. USA: Williams \& Wilkins, 1985

11. Lowery M, Nolan P, O'Maley M. Electromyogram median frequency, spectral compression and muscle fibre conduction velocity during sustained sub-maximal contraction of the brachioradialis muscle. J Electromyogr Kinesiol 2002;12:111-8.

12. Esposito F. Electromyogram and mechanomyogram changes in fresh and fatigued muscle during sustained contraction in men. Eur J Appl Physiol 1998;78:492-501.

13. Hary D, Belman MJ, Propst J, Lewis S. A statical analysis of the spectral moments used in EMG test of endurance. J Appl Physiol 1982;53:779-83.

14. Roy SH, DeLuca CJ. Fatigue, recovery and low-back pain in varsity rowers. Med Sci Sport Exerc1990;22:463-9.

15. Dias da Silva S, Gonçalves M. Dynamic and isometric protocols of knee extension: effect of fatigue on the EMG signal. Electromyogr Clin Neurophysiol 2006:35:42-6.

16. Guedes DP, Lopes CC, Guedes JERP. Reprodutibilidade e validade do Questionário Internacional de Atividade Física em adolescentes. Rev Bras Med Esporte 2005;1:151-8. determinação e diferenciação do comportamento da fadiga muscular localizada. Especificamente, a eletromiografia, através dos parâmetros analisados, demonstrou ser uma ferramenta eficaz para tal objetivo. Através da freqüência do sinal EMG pode-se diferenciar e caracterizar ambos os grupos com relação à fadiga muscular localizada.

Também pode-se constatar que as dificuldades inerentes à aplicação dessa ferramenta, em uma situação prática, são as mesmas enfrentadas por outros pesquisadores. Constitui como objetivo final para a área o desenvolvimento de métodos validados capazes de monitorar o fenômeno da fadiga muscular localizada. Entretanto, ressalta-se que essa não é uma condição sine qua non para o início da aplicação dos métodos já existentes. Permite, naturalmente, que o processamento no sinal EMG possa ser trabalhado sob diversas dimensões, por exemplo, utilizando-se parâmetros no domínio da freqüência do sinal EMG.

\section{CONCLUSÃO}

Pode-se sugerir que a conclusão deste estudo reside, simplesmente, no fato de que a análise no domínio da freqüência do sinal EMG demonstrou ser viável para a diferenciação entre sujeitos atletas e sedentários.

Entretanto, é bastante improvável que dessa conclusão resulte uma solução eficiente para a avaliação da fadiga muscular localizada. Nessa lacuna encontram-se as justificativas que servirão de base para o desenvolvimento de estudos futuros abordando essa mesma temática. Ela é controversa, conflituosa e complexa; talvez esses adjetivos expliquem a grande quantidade de estudos publicados, abordando essa temática, paradoxalmente à pouca informação conclusiva produzida.

Nesse contexto, além da conclusão já mencionada, não se pode deixar de explorar talvez o único consenso em torno desse tema: a contínua busca de um nível operacional dessa metodologia é de fundamental importância para o desenvolvimento de plataformas de avaliação com real potencial de aplicação no âmbito clínico e, principalmente, na área do treinamento esportivo.

Todos os autores declararam não haver qualquer potencial conflito de interesses referente a este artigo.

17. Ebersole KT, O'Connor KM, Wier AP. Mechanographic and electromyographic responses to repeated concentric muscle actions of quadriceps femoris. J Electromyogr Kinesiol. 2005;16:149-57.

18. Ebersole KT, Housh TJ, Johnson GO, Evetovich TK, Smith DB, Pery SR. MMG and EMG responses of de superficial quadriceps femoris muscles. J Electromyogr Kinesiol 1999;7:219-27.

19. Pincivero DM, Gandhi V, Timmons MK, Coelho AJ. Quadriceps femoris eletromyogram during concentric, isometric and eccentric phases of fatiguing dynamic knee extensions. J Biomech 2006;39:246-54.

20. Pincivero DM. Knee extensor torque and quadriceps femoris EMG during perceptually-guided isometric contractions. J Electromyogr Kinesiol 2003;13:159-67.

21. Pincivero DM. Gender and muscle differences in EMG amplitude and median frequency, and variability during maximal voluntary contractions of the quadriceps femoris. J Electromyogr Kinesio 2000;10:189-96.

22. Pompeu FAMS. Guia para estudos em biodinâmica do movimento humano. São Paulo: Ph Editora, 2006

23. Negrão Filho RF, Silva PB, Alves N, Padovani CR, Azevedo FM. Stabilization of lumbo-pelvic region and electromyography of abdominal muscles. Electromyogr Clin Neurophysiol 2006;46:51-7.

24. Branco V, Negrão Filho RF, Padovani CR, Azevedo FM, Alves N, Carvalho AC. Relação entre a tensão aplicada e a sensação de desconforto nos músculos isquiotibiais durante o alongamento. Rev Bras Fisioter 2006;10:465-72.

25. Maisetti $\mathrm{O}$, Guével A, Legros $P$, Hogrel J. SEMG power spectrum changes during a sustained $50 \%$ maximum voluntary isometric torque do not depend upon the prior knowledge of the exercise duration. J Electromyogr Kinesiol 2002;12:102-9.

26. Nicolas A, Gauthier A, Trouillet J, Davenne D. The influence of circadian rhythm during a sustained submaximal exercise and on recovery process. J Electromyogr Kinesiol 2008;18:284-90.

27. Weir JP, Beck TW, Cramer JT, Housh TJ. Is fatigue all in your head? A critical review of the central governor model. Br J Sports Med 2006;40:573-86.

28. Borg G. Psycophysical scaling with applications in physical work and the perception of exertion. Scand J Work Environ Health 1990;16:55-8.

29. Pavlat, DT, Housh TJ, Johnson GO, Eckerson JM. Electromyographic reponses at the neuromuscular fatigue threshold. J Sports Med Phys Fitness 1995;35:31-7.

30. Pavlat, DT, Housh TJ, Johnson GO, Schmidt RJ, Eckerson JM. An examination of the electromyographic fatigue threshold test. Eur J Appl Physiol Occup Physiol 1993:4:305-8.

Rev Bras Med Esporte - Vol. 14, № 6 - Nov/Dez, 2008 